\title{
Optimal Auctions with Simultaneous and Costly Participation*
}

\author{
Gorkem Celik and Okan Yilankaya
}

\begin{abstract}
We study the optimal auction problem with participation costs in the symmetric independent private values setting, where bidders know their valuations when they make independent participation decisions. After characterizing the optimal auction in terms of participation cutoffs, we provide an example where it is asymmetric. We then investigate when the optimal auction will be symmetric/asymmetric and the nature of possible asymmetries. We also show that, under some conditions, the seller obtains her maximal profit in an (asymmetric) equilibrium of an anonymous second price auction. In general, the seller can also use non-anonymous auctions that resemble the ones that are actually observed in practice.
\end{abstract}

KEYWORDS: optimal auctions, participation costs, endogenous entry, asymmetry, bidding preferences

*Earlier versions of this paper circulated under the title "Optimal Auctions with Participation Costs." Two referees and a Co-Editor provided many useful comments and suggestions, for which we are grateful. We thank seminar audiences at Bilkent University, Bogaziçi University, HEC Paris, Hong Kong University, Hong Kong University of Science and Technology, Indian Statistical Institute (Delhi Centre), Koç University, Sabanci University, The University of Adelaide, The University of British Columbia, The University of Melbourne, The University of New South Wales, The University of Texas at Austin, The University of Western Ontario, University of Southern California, and at various conferences and workshops. We thank Social Sciences and Humanities Research Council of Canada for research support. 
Celik and Yilankaya: Optimal Auctions with Simultaneous and Costly Participation

\section{Introduction}

In many auction environments bidders incur participation costs even when they know their valuations for the object being sold or how much they will bid: Bidders are sometimes required to purchase bid documents, to pre-qualify or register for the auction, or to be present at the auction site, all of which may be costly. In procurement and sales of public assets, a "bid" is often more than a dollar amount; it must also include a detailed plan with the requisite documentation. Procurement auctions usually require the posting of bid bonds by all bidders before the auction and a performance bond by the winner immediately after. There may be fixed costs associated with securing bid bonds and making arrangements in advance for performance bonds, or for financing in general in other environments.

In this paper, we study the optimal (profit-maximizing) auction problem with costly participation in the standard symmetric independent private values setting. ${ }^{1,2}$ After the seller, who owns an indivisible object, announces her selling mechanism, bidders independently decide whether to participate in it or not. ${ }^{3}$ Bidders know their valuations when they make their decisions. We model costly participation in a simple and stylized manner: Any bidder who chooses to participate incurs a real resource cost that is independent of both the seller's mechanism and her planned action in it.

We show that the search for the optimal auction need not involve considering stochastic bidder participation decisions. Each bidder will participate in the optimal auction iff her valuation is greater than a cutoff value. Given an arbitrary profile of these (bidder-specific) participation cutoffs, the optimal allocation rule is a familiar one: The bidder with the highest valuation among participants shall receive the object. The seller's problem is therefore reduced to finding the optimal participation cutoffs.

We give an example where the optimal auction is asymmetric in our symmetric environment, i.e., bidders have different cutoffs. ${ }^{4}$ We then provide a

\footnotetext{
${ }^{1}$ Bidder asymmetries do not present any conceptual difficulties. We assume that bidders are ex-ante symmetric to keep the notation simple, since we will later focus on properties of optimal auctions in a symmetric environment.

${ }^{2}$ Our results are also applicable to the efficient auction problem, as we will elaborate later.

${ }^{3}$ Our use of the term "auction" therefore is more restrictive than in Myerson (1981): We only allow mechanisms where bidders' participation decisions depend solely on their own valuations. This constraint may be binding, unlike in the standard setup, due to the existence of participation costs.

${ }^{4}$ Since the environment is symmetric, ex-ante randomization by the seller among auctions with the same set of cutoffs (with bidders' identities permuted) will restore pre-
} 
The B.E. Journal of Theoretical Economics, Vol. 9 [2009], Iss. 1 (Advances), Art. 24

sufficient condition for this to happen in general. As an immediate corollary, this result identifies valuation distribution functions for which the optimal auction is asymmetric independent of the magnitude of the participation cost $c$ and the number of bidders $n$. Note that in asymmetric auctions the object is not necessarily assigned to the highest valuation bidder (who may be a nonparticipant). The optimal auction does not have this type of allocative inefficiency when there are no participation costs. ${ }^{5}$ We then characterize distribution functions for which the optimal auction is symmetric independent of $c$ and $n$. We also provide some results about the nature of possible asymmetries that simplify the task of finding the optimal cutoffs.

We analyze the case of uniformly distributed valuations in detail, where it is possible to give a complete characterization of optimal auctions by using our previous results. In particular, depending on the support of the distribution, the optimal auction will be either symmetric or it will have only two distinct cutoffs where the smaller one is used by only one of the bidders. An interesting feature of the optimal auction is that whenever it is asymmetric the seller will exclusively deal with a single bidder, i.e., "sole-source," if the participation cost is high enough.

The implementation of asymmetric optimal auctions is the final issue we address. We show that, under some conditions, the seller will obtain her maximal profit in an (asymmetric) equilibrium of a second price auction that is anonymous, i.e., with rules that do not discriminate among bidders. In general, the seller can use first or second price auctions where some bidders are preferentially treated. ${ }^{6}$

In our model the cost incurred by participating bidders is independent of the auction chosen by the seller. Yet, in many cases, this cost is endogenous; it is the seller who requires pre-qualification, a detailed plan with documents, or bid and performance bonds. However, there are good reasons for these types of requirements that are outside of our standard models, like making sure that

randomization symmetry in a trivial sense. In this paper we study the auction that ends up being used, which the seller might have chosen through such a randomization.

${ }^{5}$ We are referring to the "regular" case of increasing virtual valuation functions with symmetric bidders. However, there is a difference also with the asymmetric bidders case: In our setup, the optimal auction does not necessarily assign the object to the bidder with the highest virtual valuation either.

${ }^{6}$ Examples include government-run auctions where domestic/in-state/small businesses are preferentially treated (see Section 3). We are not claiming that the objective of these policies is profit maximization. The examples illustrate that discrimination among bidders does happen, and that it may not be as detrimental to the seller's profit as one may have thought, even in a symmetric environment. McAfee and McMillan (1989) and Ayres and Cramton (1996) make the latter point in asymmetric environments.

http://www.bepress.com/bejte/vol9/iss1/art24 
the winner can and will do as she promises, and securing, or at least improving, the integrity of the auction process. ${ }^{7}$ The participation cost in our setup can be thought as the smallest amount necessary for running any auction as in our textbook models, where doing so is preferable to the alternatives. ${ }^{8}$

We assume that, after the seller chooses her mechanism, bidders make their participation decisions independently, and thus study a constrained problem. The class of mechanisms allowed by this assumption, which includes standard auctions and their variations, is large enough and has received considerable interest both in academia and in practice. However, it leaves out sequential participation mechanisms where a bidder's participation decision can be conditioned on participation decisions and the revealed valuations of the bidders who are contacted earlier by the mechanism. ${ }^{9}$ Note that even when such mechanisms are available, auctions with simultaneous participation may be favored because of transparency benefits, or due to the high cost of time delay, among other factors. ${ }^{10}$

There are a few papers that use our setup where bidders know their valuations when they make simultaneous costly participation decisions. ${ }^{11}$ Samuelson (1985) shows that both ex-ante total surplus and the seller's revenue may decline with the number of bidders $n$ in symmetric equilibria of first price

\footnotetext{
${ }^{7}$ The last one may be critically important when an agent must run the auction for the principal, which is the case for government procurement or sales of public assets. This issue is also relevant when comparing auctions to private negotiations.

${ }^{8}$ Note that the seller would like the participation cost to be as small as possible in our setup.

${ }^{9}$ Ehrman and Peters (1994) provide the following formalization: They assume that the seller contacts the bidders in a pre-determined (by nature) sequence unobservable to bidders. Each time the seller meets an additional bidder, she sends a costless binary signal revealing only whether she is willing to negotiate with this bidder or not. After receiving this signal, the bidder updates her belief on the valuations of the bidders who are already contacted by the seller and then decides whether to incur the participation cost.

${ }^{10}$ For example, the general rule for government procurement in the US, as well as in many other countries, is "full and open competition," see the Federal Acquisition Regulation.

${ }^{11}$ There is another strand of literature where costly entry, or information acquisition, decisions are made ex ante. See, among others, Matthews (1984), McAfee and McMillan (1987), Harstad (1990), Tan (1992), Engelbrecht-Wiggans (1993), Levin and Smith (1994), Persico (2000), and Bergeman and Valimaki (2002). Compte and Jehiel (2007) study dynamic formats, e.g., ascending price auctions, that allow bidders to observe the number of competitors remaining when they make costly information acquisition decisions. In a similar vein, McAfee and McMillan (1988) and Cremer et al. (2007) consider sequential (costly) search mechanisms where the seller incurs a cost when she invites an additional bidder to the mechanism. In all these papers, the players who make costly entry/search decisions do not have any private information. In contrast, we consider an environment where each bidder's participation decision can be conditioned on her privately known valuation.
} 
auctions with reserve prices, which are chosen optimally (given the respective criterion) for fixed $n .^{12}$

Stegeman (1996) studies ex-ante efficient auctions (maximizing social surplus). He shows that the efficient auction is characterized by participation cutoffs and provides an example where it is asymmetric. He also shows that the second price auction always has an efficient equilibrium, whereas the first price auction has one iff the symmetric equilibrium of the second price auction is efficient. Our paper differs from Stegeman's (1996) in that we consider optimal auctions, which necessitates an independent proof for the characterization result in term of cutoffs. ${ }^{13}$ More importantly, we investigate the conditions under which the optimal auction will be symmetric, the nature of possible asymmetries, and the implementation question.

At this juncture, we would like to point out that our results about the properties of optimal cutoffs are pertinent in the efficient auction problem as well. In particular, corresponding results for efficient auctions can be obtained via a simple substitution in our results, which we will identify after the formal analysis. Therefore, our paper also provides a contribution in terms of efficient auctions, complementing Stegeman's (1996).

Campbell (1998) gives a sufficient condition for existence of asymmetric equilibria in second price auctions when there are two bidders. He shows that, under some conditions, the bidders benefit from coordinating on the most asymmetric of these equilibria via a correlating device with publicly observable signals, and that preplay communication will help even more.

Finally, we benefited significantly from the methods used by a related paper by Tan and Yilankaya (2006) who study equilibria of second price auctions and provide sufficient conditions for uniqueness and multiplicity.

The rest of the paper is organized as follows: We study optimal auctions in Section 2 and how to implement them in Section 3. All the proofs, except that of Lemma 1, are in the Appendix.

\footnotetext{
${ }^{12}$ His finding also applies to any symmetric and increasing equilibrium of any anonymous auction where the highest bidder receives the object. Menezes and Monteiro (2000) and $\mathrm{Lu}$ (2009) show that the auctions considered by Samuelson (1985) are optimal if the seller is restricted to consider symmetric equilibria of anonymous auctions. Lu (2009) concludes from this observation that the seller's maximized revenue within this class of equilibria may decline in $n$, pointing to the possibility that the unrestricted optimal auction is asymmetric.

${ }^{13}$ Transfers from bidders to the seller, which is of paramount importance for the optimal auction problem, do not affect the social surplus.
} 
Celik and Yilankaya: Optimal Auctions with Simultaneous and Costly Participation

\section{Optimal Auctions}

\subsection{The Environment}

We consider a symmetric independent private values environment. There is a risk-neutral seller who wants to sell an indivisible object for which her valuation is zero. There are $n \geq 2$ risk-neutral potential buyers, or "bidders." Let $v_{i}$ denote the valuation of bidder $i \in N=\{1, \ldots, n\}$. Bidders' valuations are independently distributed according to the cumulative distribution function $F(\cdot)$ with continuously differentiable and positive density $f(\cdot)$ on $\left[v_{l}, v_{h}\right]$, where $0 \leq v_{l}<v_{h}<\infty$. Bidders know their own valuations. All of this is common knowledge. We assume throughout that the virtual valuation function, i.e., $J(v)=v-\frac{1-F(v)}{f(v)}$, is increasing. ${ }^{14}$ The seller's problem is to choose an auction mechanism (to which we assume she can commit) that maximizes her expected profit in one of its Bayesian-Nash equilibria. ${ }^{15}$

We depart from this standard optimal auction problem in two ways. The first is "costly participation." Any bidder who chooses to participate in the seller's auction incurs a commonly known real resource cost of $c \in\left(0, v_{h}\right)$. Note that this cost is independent of both the particular auction chosen by the seller and the bidder's (planned) behavior in it. We also assume that nonparticipating bidders neither receive the object nor make any payments. ${ }^{16}$ The second difference is that we consider a restricted class of mechanisms in that bidders make independent participation decisions. At the time of these decisions, bidders already possess their private information, i.e., they know their own valuations.

A few remarks are in order. We rule out agent-to-agent communication with our independent participation assumption. ${ }^{17}$ We do not allow, for example, the seller to communicate with only one of the bidders, and let her freely learn the valuations of other bidders. Note that the lack of communication among agents is a sufficient condition for our assumption that bidders behave

\footnotetext{
${ }^{14}$ Myerson (1981) shows how to dispense with this standard regularity assumption.

${ }^{15}$ In what follows we investigate the properties of the mechanism that is communicated to the bidders which the seller may have chosen through an ex-ante randomization, see Footnote 4.

${ }^{16}$ Stegeman (1996) calls this the "no passive reassignment rule." Note that it may be seen as a consequence of the costly participation issue we are addressing: Voluntarily receiving the object (a premise we maintain throughout) negates the idea of nonparticipation.

${ }^{17}$ See McAfee and McMillan (1988) for a detailed and very useful discussion of these issues. In their terminology, we restrict attention to principal-centered mechanisms (without any agent-to-agent communication), just as they do.
} 
The B.E. Journal of Theoretical Economics, Vol. 9 [2009], Iss. 1 (Advances), Art. 24

noncooperatively in the seller's auction, which is a standard premise in the optimal auction literature. ${ }^{18}$

The independent participation assumption does not preclude dynamic auctions, such as English or Dutch formats, where participating bidders observe the set of participants (or, more generally, some noisy signal about it), as long as the (costly) participation decisions are made before the (costless) bidding process starts. Note, however, that the seller does not gain anything by considering dynamic auctions of this kind. That would only introduce additional (incentive) constraints to the problem in which bidders simultaneously make participation and "bidding" decisions. ${ }^{19}$ Moreover, it turns out that the seller does not lose anything either if bidders were to observe the set of participants and update their beliefs about them: As we will discuss in Section 3, a second price auction is optimal in our (unconstrained) problem, and since its equilibrium is in dominant strategies, what the bidders know about the set of participants is immaterial.

\subsection{Optimal Auction up to Participation Cutoffs}

In this section we will show that the seller can restrict attention to those with deterministic participation decisions when searching for optimal auctions. ${ }^{20}$ In particular, each bidder will participate in the optimal auction iff her valuation is greater than her participation cutoff. Once we fix these bidder-specific cutoffs the seller's problem becomes identical to that in the standard environment, i.e., $c=0$, except the requirement that nonparticipating types do not receive the object. Therefore, the solution is similar as well: The bidder with the highest valuation among participants will receive the object (Lemma 1). After this characterization of the optimal allocation rule given arbitrary participation cutoffs, we investigate the optimal cutoffs and present our main results in Section 2.3.

Consider any (Bayesian-Nash) equilibrium of any auction that the seller may choose. Since bidder $i$ is risk-neutral, she cares only about her expected probability of winning the object, denoted by $Q_{i}$, and her expected payment,

${ }^{18}$ The seller obviously does not want the bidders to collude, everything else being constant, but she would save in terms of participation costs if bidders were able to communicate freely among themselves.

${ }^{19}$ Therefore, without any loss of generality (in terms of finding the optimal auction), the setup we consider can be represented as follows: The bidders simultaneously choose messages from $\left\{N_{o}\right\} \cup\left[v_{l}, v_{h}\right]$, where $N_{o}$ (denoting nonparticipation) is free and all other messages cost $c$. The seller's mechanism consists of assignment and transfer rules that map message profiles. Bidders who send No receive the object with probability zero.

${ }^{20}$ This is not necessarily true for arbitrary auctions. Optimality is crucial in our argument. 
Celik and Yilankaya: Optimal Auctions with Simultaneous and Costly Participation

denoted by $P_{i}$. Notice two differences from the standard setup: $Q_{i}$ incorporates $i$ 's probability of participating in the auction, denoted by $\rho_{i}$, and $P_{i}$ incorporates the expected participation cost that $i$ incurs. The equilibrium expected payoff of type- $v_{i}$ bidder $i$ ( $v_{i}$ for short) can thus be written as

$$
\pi_{i}\left(v_{i}\right)=Q_{i}\left(v_{i}\right) v_{i}-P_{i}\left(v_{i}\right)
$$

It must be the case that $v_{i}$ does not want to imitate the equilibrium behavior of any $v_{i}^{\prime}$, which consists of $v_{i}^{\prime}$ 's participation decision $\rho_{i}\left(v_{i}^{\prime}\right)$ as well as her actions in the auction when she participates. Using standard arguments, this implies that

$$
\pi_{i}\left(v_{i}\right)=\pi_{i}\left(v_{l}\right)+\int_{v_{l}}^{v_{i}} Q_{i}(y) d y
$$

and that $Q_{i}(\cdot)$ is weakly increasing. ${ }^{21,22}$

However, in our setup, where bidders have full control of the participation decisions that they make, we also need to make sure that $v_{i}$ does not have an incentive to choose any participation probability, not only $\rho_{i}\left(v_{i}^{\prime}\right)$. This will indeed be the case for the optimal auction for which all participation decisions can be taken to be deterministic, without any loss of generality.

The seller's expected profit (also revenue, since her valuation is zero) is

$$
\pi_{s}=\sum_{i \in N}\left\{\int_{v_{l}}^{v_{h}}\left[J\left(v_{i}\right) Q_{i}\left(v_{i}\right)-\rho_{i}\left(v_{i}\right) c\right] f\left(v_{i}\right) d v_{i}-\pi_{i}\left(v_{l}\right)\right\}
$$

where the term in braces is bidder $i$ 's expected payment to the seller, calculated by using (1), (2), and the fact that the participation cost is incurred by bidders, but not received by the seller.

In the optimal auction, the lowest type of each bidder will obtain zero equilibrium expected payoff, i.e., $\pi_{i}\left(v_{l}\right)=0, \forall i \in N$. Moreover, for each $i$, since $Q_{i}(\cdot)$ is weakly increasing, there exists a cutoff $\widetilde{v}_{i} \in\left[v_{l}, v_{h}\right]$ such that $Q_{i}\left(v_{i}\right)=0$ for $v_{i}<\widetilde{v}_{i}$ and $Q_{i}\left(v_{i}\right)>0$ for $v_{i}>\widetilde{v}_{i}$. It follows from (2) that $\pi_{i}\left(v_{i}\right)=0$ for $v_{i} \leq \widetilde{v}_{i}$ and $\pi_{i}\left(v_{i}\right)>0$ for $v_{i}>\widetilde{v}_{i}$. This in turn implies that

\footnotetext{
${ }^{21}$ Note that $\pi_{i}(\cdot)$ is also weakly increasing, and it is increasing whenever $Q_{i}(\cdot)>0$.

${ }^{22}$ Suppose that the seller's auction is dynamic (e.g., English or Dutch formats) in the sense that bidders first make their participation decisions and then bid after observing the set of participants (or a signal about it) and updating their priors about other bidders. Condition (2) is still necessary, as it is obtained by "averaging" the incentive constraints over all possible signals that bidder $i$ can observe in equilibrium. We do not need to characterize the sufficient conditions, since they will be just additional constraints in the optimal auction problem. However, as we will show in Section 3, they are nonbinding anyway.
} 
The B.E. Journal of Theoretical Economics, Vol. 9 [2009], Iss. 1 (Advances), Art. 24

bidders' participation decisions will be deterministic for almost all types for the optimal auction. To see this, first note that $\rho_{i}\left(v_{i}\right)=0$ for all but a measure zero set of $v_{i}<\widetilde{v}_{i}$ : We have $Q_{i}\left(v_{i}\right)=\pi_{i}\left(v_{i}\right)=0$ for $v_{i}<\widetilde{v}_{i}$. If a positive measure set of these types were participating in an auction, then the seller can simply save the participation costs that must be incurred to induce their participation without affecting others' incentives. ${ }^{23,24}$ Secondly, we have $\rho_{i}\left(v_{i}\right)=1$ for all $v_{i}>\widetilde{v}_{i}$, which follows from these types' optimal participation decisions: Since their overall payoff is strictly positive, their payoff from participation must be strictly positive as well (notice that payoff from nonparticipation is zero). Combining these two steps, we conclude that bidder $i$ will participate in the optimal auction with probability one (respectively, zero) if her valuation is greater (respectively, less) than $\widetilde{v}_{i} \in\left[v_{l}, v_{h}\right] .{ }^{25}$ Incorporating these deterministic participation decisions and $\pi_{i}\left(v_{l}\right)=0, \forall i \in N$ into (3), we have

$$
\pi_{s}=\sum_{i \in N} \int_{v_{l}}^{v_{h}} J\left(v_{i}\right) Q_{i}\left(v_{i}\right) f\left(v_{i}\right) d v_{i}-c \sum_{i \in N}\left[1-F\left(\widetilde{v}_{i}\right)\right],
$$

with $Q_{i}\left(v_{i}\right)=0$ for $v_{i}<\widetilde{v}_{i}$. Let $q_{i}\left(v_{1}, \ldots, v_{n}\right)$ be $i$ 's equilibrium probability of winning the object when the valuations are $\left(v_{1}, \ldots, v_{n}\right)$. Since

$$
Q_{i}\left(v_{i}\right)=\int_{0}^{1} \ldots \int_{0}^{1} q_{i}\left(v_{1}, \ldots, v_{n}\right) \prod_{\substack{j \neq i \\ j \in N}} f\left(v_{j}\right) d v_{j},
$$

\footnotetext{
${ }^{23}$ Suppose we modify the auction so that types of bidder $i$ who participate but have zero probability of receiving the object are not participating in the new one. There is no change in $Q_{i}(\cdot)$. Moreover, we can construct an allocation rule for the new auction that does not change any $Q_{j}(\cdot), j \neq i$ either, even when bidder $i$ 's participation made a difference to other bidders. For instance, consider an auction where bidder 2 never receives the object, bidder 1 receives the object if and only if bidder 2 participates, and all types of bidder 2 participate with probability $\rho>0$. We modify this auction by excluding all types of bidder 2 and assigning the object to bidder 1 with probability $\rho$ : Incentives for bidder 1 do not change and the seller saves on the cost she must incur to induce bidder 2's participation in the old auction.

${ }^{24}$ In what follows we will let $\rho_{i}\left(v_{i}\right)=0$ for all $v_{i}<\widetilde{v}_{i}$. Clearly, this is without loss of generality in terms of expected payoffs of the bidders and the seller.

${ }^{25}$ Note that if it is not profitable for $v_{i}$ to imitate any $v_{i}^{\prime}$ (inclusive of $\rho_{i}\left(v_{i}^{\prime}\right) \in\{0,1\}$ ), then it will not be profitable for $v_{i}$ to use a nondegenerate participation probability (and then imitate the action of $v_{i}^{\prime}$ in the auction), since this will yield an expected payoff which is just a convex combination of what $v_{i}$ would receive if she were to imitate $v_{i}^{\prime}$ and the payoff from nonparticipation.
} 
Celik and Yilankaya: Optimal Auctions with Simultaneous and Costly Participation

we can rewrite the seller's expected profit as

$$
\pi_{s}=\int_{v_{l}}^{v_{h}} \ldots \int_{v_{l}}^{v_{h}}\left[\sum_{i \in N} J\left(v_{i}\right) q_{i}\left(v_{1}, \ldots, v_{n}\right)\right] \prod_{i=1}^{n} f\left(v_{i}\right) d v_{i}-c \sum_{i \in N}\left[1-F\left(\widetilde{v}_{i}\right)\right] .
$$

It is useful to think the seller's problem in two steps. We first find equilibrium winning probabilities that maximize the seller's expected profit for fixed cutoffs. $^{26}$ We then turn our attention to optimal cutoffs in Section 2.3.

For the first step, consider arbitrary cutoffs where virtual valuations are nonnegative. $^{27}$ The following notation will be useful throughout the paper. Let $v_{0} \in\left[v_{l}, v_{h}\right]$ be the smallest valuation for which the virtual valuation is nonnegative. That is, if $J\left(v_{l}\right)<0$, then $v_{0} \in\left(v_{l}, v_{h}\right)$ is given by $J\left(v_{0}\right)=0$; if $J\left(v_{l}\right) \geq 0$, then $v_{0}=v_{l}$. (Note that $J(\cdot)$ is increasing and $J\left(v_{h}\right)=v_{h}>0$.)

Given the cutoffs, the seller's problem is to maximize (6) with respect to $q_{i}(\cdot)$ 's subject to the constraints that these are probabilities, they indeed imply the given cutoffs, nonparticipating bidders neither obtain the object nor affect any participating bidder's probability of obtaining the object, and the induced expected winning probabilities are weakly increasing. That is, given $\widetilde{v}_{i}$ 's, $q_{i}\left(v_{1}, \ldots, v_{n}\right)$ (and the resulting $Q_{i}\left(v_{i}\right)$ 's) must satisfy the following constraints for all $i \in N,\left(v_{1}, \ldots, v_{n}\right) \in\left[v_{l}, v_{h}\right]^{n}$, and $v_{i}, v_{i}^{\prime} \in\left[v_{l}, v_{h}\right]$ :

- $q_{i}\left(v_{1}, \ldots, v_{n}\right) \geq 0$ and $\sum_{i \in N} q_{i}\left(v_{1}, \ldots, v_{n}\right) \leq 1$.

- $q_{i}\left(v_{1}, \ldots, v_{n}\right)=0$ for $v_{i}<\widetilde{v}_{i}$ and $q_{i}\left(v_{1}, \ldots, v_{j}, \ldots v_{n}\right)=q_{i}\left(v_{1}, \ldots, v_{j}^{\prime}, \ldots v_{n}\right)$ for all $j \in N$ and $v_{j}, v_{j}^{\prime}<\widetilde{v}_{j}$.

- $Q_{i}\left(v_{i}\right)>0$ for $v_{i}>\widetilde{v}_{i}{ }^{28}$

- $Q_{i}\left(v_{i}\right) \geq Q_{i}\left(v_{i}^{\prime}\right)$ for $v_{i}>v_{i}^{\prime}$.

Notice that the seller's problem is identical to that of the standard optimal auction setup $(c=0)$, except that participation cutoffs of the bidders must be respected. ${ }^{29}$ Maximizing (6) pointwise results in the object being assigned with

\footnotetext{
${ }^{26}$ Notice that equilibrium winning probabilities and cutoffs are related: $q_{i}(\cdot)$ determines $Q_{i}(\cdot)$ (see $(5)$ ), which in turn determines $\widetilde{v}_{i}$. Therefore, our search for $q_{i}(\cdot)$ 's for fixed $\widetilde{v}_{i}$ 's is constrained to be among those that would imply these $\widetilde{v}_{i}$ 's.

${ }^{27}$ Notice that this is indeed the case for optimal cutoffs: The seller is better off not selling to negative virtual types.

${ }^{28}$ We also need $Q_{i}\left(v_{i}\right)=0$ for $v_{i}<\widetilde{v}_{i}$, but this is implied by the second constraint.

${ }^{29}$ Given cutoffs, total participation cost incurred is fixed, and hence plays no role.
} 
The B.E. Journal of Theoretical Economics, Vol. 9 [2009], Iss. 1 (Advances), Art. 24

positive probability only to bidders who have the highest virtual valuations, and hence valuations, among participants. ${ }^{30}$

We have characterized the optimal auction up to the level of participation cutoffs, which we summarize next.

Lemma 1 In the optimal auction there exists a cutoff point for each bidder such that she participates in the auction if and only if her valuation is greater than her cutoff, i.e., $\forall i \exists \widetilde{v}_{i} \geq v_{0}$ such that $\rho_{i}\left(v_{i}\right)=0$ (hence $Q_{i}\left(v_{i}\right)=\pi_{i}\left(v_{i}\right)=$ $0)$ for $v_{i}<\widetilde{v}_{i}$ and $\rho_{i}\left(v_{i}\right)=1$ for $v_{i}>\widetilde{v}_{i}$. For each $\left(v_{1}, \ldots, v_{n}\right)$ the equilibrium winning probabilities satisfy:

i) If $v_{j}<\widetilde{v}_{j} \forall j \in N$, then $q_{i}\left(v_{1}, \ldots, v_{n}\right)=0 \quad \forall i \in N$. If $\exists j$ s.t. $v_{j}>\widetilde{v}_{j}$, then $\sum_{i \in N} q_{i}\left(v_{1}, \ldots, v_{n}\right)=1$.

ii) $q_{i}\left(v_{1}, \ldots, v_{n}\right)>0 \Rightarrow v_{i} \geq v_{j} \forall j \in N$ s.t. $v_{j} \geq \widetilde{v}_{j}$.

Remark 1 (Revenue Equivalence) Consider two auctions, say $A$ and $B$, that, in equilibrium, assign the object to the highest-valuation participant and have the same participation cutoff for each bidder, i.e., $\widetilde{v}_{i}^{A}=\widetilde{v}_{i}^{B} \forall i \in N$ (with the associated cutoff rule in participation we discussed above), where expected payoffs of the marginal types are equal as well, i.e., $\pi_{i}\left(\widetilde{v}_{i}^{A}\right)=\pi_{i}\left(\widetilde{v}_{i}^{B}\right) \forall i \in N$. The expected payoff of every type of every bidder, and hence that of the seller, is the same in both auctions.

\subsection{Optimal Participation Cutoffs}

We now turn our attention to optimal cutoffs. For this purpose, we first express the seller's expected profit in terms of solely bidders' participation cutoffs, utilizing what we know about optimal auctions (Lemma 1). We show with an example that the optimal auction may be asymmetric, i.e., not all bidders have identical cutoffs in our symmetric environment. We then identify a sufficient condition for the optimal auction to be asymmetric given the number of bidders $n$, the participation $\operatorname{cost} c$, and the distribution function of the valuations $F(\cdot)$ (Proposition 1). As a corollary, this result gives a condition on $F(\cdot)$ under which the optimal auction will be asymmetric for all $c$ and $n$. We next provide a characterization result for the symmetry of the optimal auction for all $c$ and $n$ (Proposition 2). Finally, we present some results about the nature of possible asymmetries that will also simplify the task of finding optimal cutoffs (Proposition 3). Taken together, these results enable us to completely

\footnotetext{
${ }^{30}$ If bidders are ex-ante asymmetric, the object will still be assigned to the bidder with the highest virtual valuation (who may not have the highest valuation anymore).
} 
Celik and Yilankaya: Optimal Auctions with Simultaneous and Costly Participation

characterize optimal auctions in some cases, e.g., when bidders' valuations are uniformly distributed.

We start with indexing the set of bidders with respect to their participation cutoffs so that

$$
v_{0} \leq \widetilde{v}_{1} \leq \widetilde{v}_{2} \leq \ldots \leq \widetilde{v}_{n} \leq v_{h}
$$

We adopt the convention that $\widetilde{v}_{n+1}=v_{h}$. Recall that in the optimal auction the object is assigned to the bidder who has the highest valuation among participants (we can ignore ties). Consider an arbitrary bidder $i$ with valuation $v$ who is a participant, i.e., with $v>\widetilde{v}_{i}$. For her to receive the object in the optimal auction, all other participating bidders must have valuations less than $v$. This means that bidders whose cutoffs are lower than $v$ need to have valuations lower than $v$. Bidders with cutoffs higher than $v$ on the other hand, need to have valuations lower than their respective cutoffs, not $v$. Therefore, bidder $i$ 's probability of receiving the object in the optimal auction is given by

$$
Q_{i}(v)=F(v)^{j-1} \prod_{k=j+1}^{n+1} F\left(\widetilde{v}_{k}\right) \text { if } \widetilde{v}_{j} \leq v \leq \widetilde{v}_{j+1}
$$

for $v>\widetilde{v}_{i}$, with $Q_{i}(v)=0$ for $v<\widetilde{v}_{i}$. Notice that, for any pair of bidders, the probability of winning functions differ at only those valuations for which only one of them is a participant: For any $i$ and $j$ with $\widetilde{v}_{i}>\widetilde{v}_{j}, Q_{i}(v)=Q_{j}(v)$ for $v>\widetilde{v}_{i}$ or $v<\widetilde{v}_{j}$, and $Q_{j}(v)>Q_{i}(v)=0$ for $v \in\left(\widetilde{v}_{j}, \widetilde{v}_{i}\right)$.

Using these probability of winning functions and (4), the expected profit of the seller can be expressed solely as a function of the cutoffs (suppressing the dependence on exogenous variables):

$\pi_{s}\left(\widetilde{v}_{1}, \ldots, \widetilde{v}_{n}\right)=\sum_{i \in N} i \int_{\widetilde{v}_{i}}^{\widetilde{v}_{i+1}} J(v)\left[F(v)^{i-1} \prod_{k=i+1}^{n+1} F\left(\widetilde{v}_{k}\right)\right] f(v) d v-c \sum_{i \in N}\left(1-F\left(\widetilde{v}_{i}\right)\right)$.

The seller's problem is thus reduced to choosing a cutoff for each bidder to maximize $\pi_{s}\left(\widetilde{v}_{1}, \ldots \widetilde{v}_{n}\right)$, which is continuous, subject to the ranking constraint of the cutoffs, i.e., (7), defining a nonempty and compact constraint set. Therefore, a solution exists.

Let $\widetilde{v}_{i}^{*}$ denote the optimal $\widetilde{v}_{i}$. Note that the maximized expected revenue of the seller increases as $c$ decreases, since the seller can choose the same cutoffs and claim the participation cost savings as direct revenue. ${ }^{31}$ Similarly,

\footnotetext{
${ }^{31}$ Note that at least one of the bidders will participate in the optimal auction with positive probability, i.e., at least one of the cutoffs will be less than $v_{h}$.
} 
The B.E. Journal of Theoretical Economics, Vol. 9 [2009], Iss. 1 (Advances), Art. 24

an exogenous increase in $n$ weakly increases the seller's maximized expected revenue, since the seller has the option to restrain newcomers' participation in the auction (by setting their cutoffs as $v_{h}$ ). ${ }^{32}$

If there are no participation costs, the optimal auction is symmetric: The object is assigned to the bidder with the highest valuation as long as her virtual valuation is positive, i.e., $\widetilde{v}_{i}^{*}=v_{0} \forall i \in N$ (Myerson, 1981).

In our setup where participation is costly the seller's profit maximization problem always admits a symmetric critical point, i.e., the first order necessary conditions for this problem are satisfied at $\widetilde{v}_{i}=v^{s} \forall i \in N$, where

$$
J\left(v^{s}\right) F\left(v^{s}\right)^{n-1}=c .
$$

This condition has a straightforward interpretation. Suppose all the bidders have cutoff $v^{s}$. Increasing the cutoff of one of the bidders slightly will decrease the gross profit of the seller by $J\left(v^{s}\right) F\left(v^{s}\right)^{n-1}$ (losing $J\left(v^{s}\right)$, the virtual valuation, when all the others' valuations are less than $v^{s}$, i.e., with probability $F\left(v^{s}\right)^{n-1}$ ), while saving her $c$, the marginal cost of inducing participation. ${ }^{33}$

The symmetric cutoff is uniquely determined, and we have $v_{0}<v^{s}<$ $v_{h}$. The existence or the uniqueness of this symmetric critical point does not depend on the data of the problem, namely $F(\cdot), c$, and $n$, but, naturally, its magnitude does.

If the seller is restricted to use a symmetric auction, it is easy to show that $\widetilde{v}_{i}=v^{s} \forall i \in N$, is indeed the solution to her profit maximization problem. ${ }^{34}$ For this reason, we call $v^{s}$ the optimal symmetric cutoff.

We want to remark at this point the connection between the optimal and efficient (maximizing ex-ante social surplus) auction problems. Stegeman (1996) shows that the efficient auction in this setup is characterized by participation cutoffs (with the associated allocation rule) as well. Given this observation, the efficient auction problem also reduces to the problem we are studying, once we replace $J(v)$ (virtual valuations, or "marginal revenue") by $v$ (valuations, or "marginal social surplus") in (9), and hence in (10). Therefore, with only this substitution, our results about optimal auctions are directly applicable to efficient auctions. ${ }^{35}$

\footnotetext{
${ }^{32}$ As we will show in Section 2.4, sometimes there will be "sole-sourcing" in the optimal auction, and increasing $n$ will have no impact on the seller's revenue.

${ }^{33}$ These are normalized (by dividing by the density) marginal gross profit and the marginal cost. The marginal profit is given by $-J\left(v^{s}\right) F\left(v^{s}\right)^{n-1} f\left(v^{s}\right)+c f\left(v^{s}\right)$.

${ }^{34}$ This does not mean that the seller cannot do better in an asymmetric equilibrium of an anonymous auction. See Section 3.

${ }^{35}$ Naturally, $v_{0}$ becomes irrelevant in this case, and so should be replaced by $v_{l}$ in the results.
} 
Celik and Yilankaya: Optimal Auctions with Simultaneous and Costly Participation

Returning to our problem, we first show that the optimal auction may be asymmetric:

Example 1 There are two bidders whose valuations are distributed according to $F(v)=v^{4}$ on $[0,1]$, and the participation cost is 0.4 .

It turns out that, for this example, the optimal auction is asymmetric. The optimal cutoffs are $\widetilde{v}_{1}^{*} \approx .816$ and $\widetilde{v}_{2}^{*} \approx .92$, yielding a profit of .2525 for the seller. If we impose symmetry, however, the seller's profit decreases to .25155 (with the optimal symmetric cutoff $v^{s} \approx$.868). Notice the allocative inefficiency of the optimal auction that we mentioned before. When the valuations of both bidders are between $\widetilde{v}_{1}^{*}$ and $\widetilde{v}_{2}^{*}$, the first bidder will obtain the object even when her valuation is less than that of the second bidder.

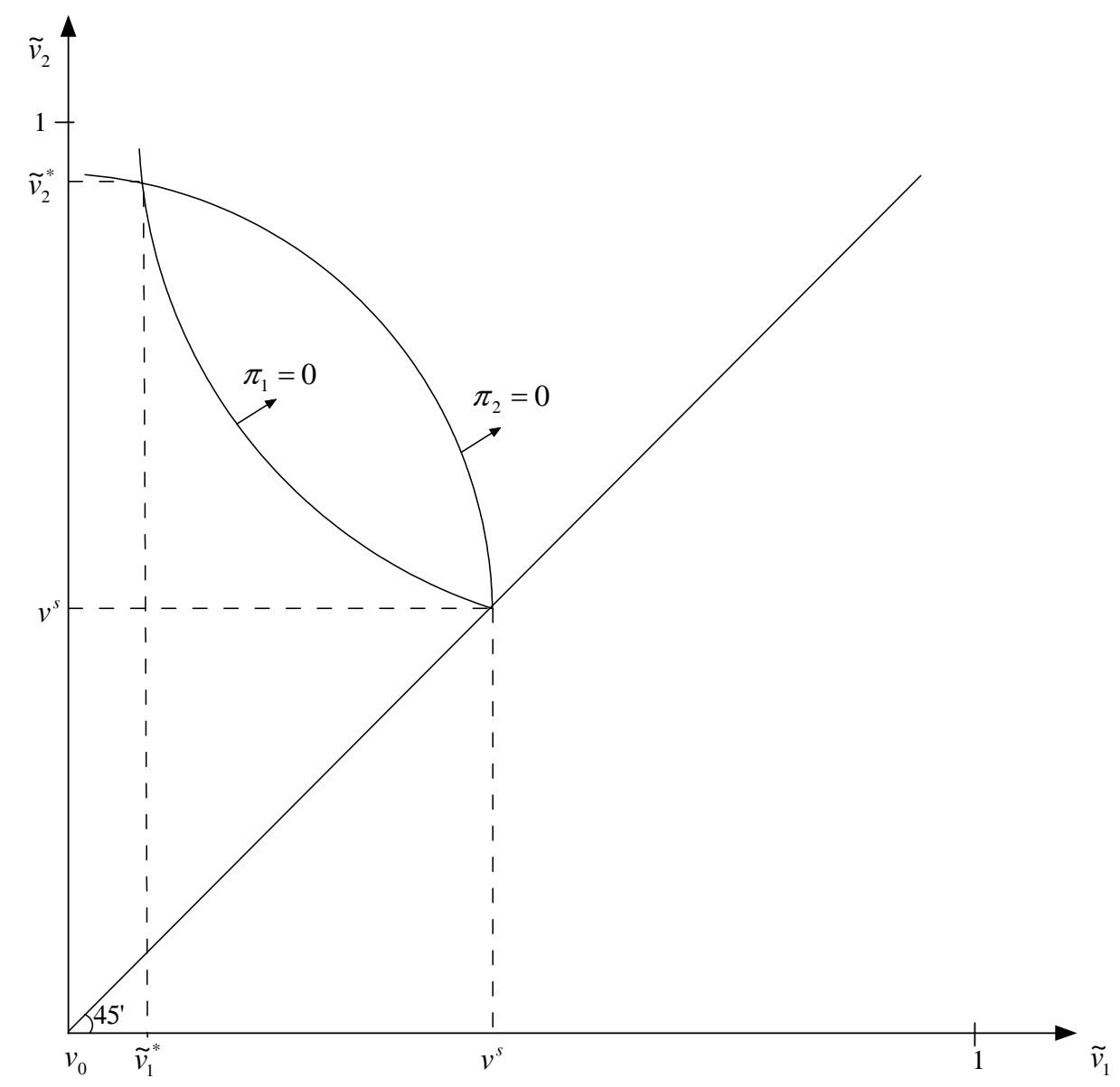

Figure 1 
We use Figure 1 not only to explain why the optimal auction is asymmetric for this example, but also to provide some pictorial intuition for Proposition 1 below and its proof. Let $\pi_{i}$ denote the marginal profit of the seller with respect to bidder $i$ 's cutoff, i.e., $\pi_{i}=\frac{\partial \pi_{s}\left(\widetilde{v}_{1}, \widetilde{v}_{2}\right)}{\partial \widetilde{v}_{i}}, i=1,2$. First order necessary conditions for optimality are satisfied, i.e., $\pi_{1}=\pi_{2}=0$, at two points: $\left(v^{s}, v^{s}\right)$ and $\left(\widetilde{v}_{1}^{*}, \widetilde{v}_{2}^{*}\right)$. However, $\left(v^{s}, v^{s}\right)$ is not even a local maximizer. At any point to the right (respectively, left) of the $\pi_{1}=0$ curve, the seller can increase her profit by decreasing (respectively, increasing) the first bidder's cutoff while keeping the second bidder's cutoff constant. ${ }^{36}$ Similar arguments apply for the second bidder's cutoff above and below the $\pi_{2}=0$ curve. Therefore, starting from the optimal symmetric cutoffs $\left(v^{s}, v^{s}\right)$, decreasing $\widetilde{v}_{1}$ while simultaneously increasing $\widetilde{v}_{2}$ by an appropriate amount, i.e., moving inside the lens-shaped area, will increase the seller's profit. ${ }^{37}$

From this discussion, it is clear that the existence of such a lens-shaped area emanating from $\left(v^{s}, v^{s}\right)$ in the admissible side of the constraint boundary (where $\widetilde{v}_{2} \geq \widetilde{v}_{1}$ ) is a sufficient condition for the suboptimality of symmetric cutoffs, which gives us our next result.

Proposition 1 (ASYMMETRY) If $\frac{J(v)}{F(v)}$ is decreasing at the optimal symmetric cutoff $v^{s}$, then the optimal auction is asymmetric. Moreover, for every $k \in\{1,2, \ldots, n-1\}$, there is an auction where $k$ bidders use one cutoff $\widetilde{v}_{i}=a<v^{s}$ for $\left.i=1, \ldots, k\right)$ and the remaining bidders use another one $\widetilde{v}_{i}=b>v^{s}$ for $\left.i=k+1, \ldots, n\right)$ that gives the seller higher profit than the optimal symmetric auction $\left.\widetilde{v}_{i}=v^{s} \forall i \in N\right)$.

We prove Proposition 1 (in the Appendix) by showing that, starting from the optimal symmetric cutoffs, the seller can increase her profits by decreasing an arbitrary group of bidders' cutoffs and increasing the cutoffs of the complementary set of bidders, as long as $\frac{J(v)}{F(v)}$ is decreasing. In other words, if $\frac{J(v)}{F(v)}$ is decreasing at $v^{s}$, then a lens-shaped improvement area, like that of Figure 1, will exist for any partition of bidders into two groups.

In order to gain some understanding of why asymmetry may be beneficial to the seller, consider the optimal symmetric cutoffs in the two-bidders case. Suppose we decrease the first bidder's cutoff slightly and increase the second bidder's cutoff so that the total expected participation cost incurred remains the same. This has an obvious negative effect: The object is sometimes sold to a low (virtual) valuation bidder 1 instead of a high valuation bidder 2

\footnotetext{
${ }^{36}$ Note that we have $\pi_{11}<0$, using the standard notation for second derivatives.

${ }^{37}$ The optimal cutoffs are indeed given by $\left(\widetilde{v}_{1}^{*}, \widetilde{v}_{2}^{*}\right)$, where the second order sufficient conditions are satisfied, as can also be seen in Figure 1.
} 
(when she is not participating). However, there is also a positive effect: Total probability of selling the object increases. ${ }^{38}$ Our sufficient condition ensures that this positive effect outweighs the negative one.

It seems that these intuitive arguments should also work when $c=0$, but we know that the optimal auction is symmetric in this case. What is special about positive $c$ ? When $c=0$ the virtual valuation is zero at the optimal symmetric cutoff. ${ }^{39}$ Therefore, decreasing a bidder's cutoff results in selling the object to her even when her virtual valuation is negative, reducing the seller's revenue, see (6). In contrast, the virtual valuation for the bidder with the lower cutoff is still positive when $c>0$.

An asymmetric auction does not always assign the object to the bidder with the highest valuation, causing allocative inefficiency. If there are no participation costs, the optimal auction will have this type of inefficiency only when bidders are heterogenous. However, even in that case the object is always assigned to the bidder with the highest virtual valuation, unlike the solution in our setup. ${ }^{40}$

When there are more than two bidders, Proposition 1 goes further than identifying a sufficient condition for the suboptimality of symmetric cutoffs. It shows that, whenever this condition is satisfied, even an arbitrary classification of the bidders into only two groups and implementation of a different cutoff for each group would improve over the optimal symmetric outcome. We find this observation relevant for analyzing the performance of auctions where one group of bidders receives preferential treatment from the seller. For example, domestic firms are sometimes given a price preference in government procurement (see McAfee and McMillan, 1989), and minority and women owned businesses received bidding credits and guaranteed financing in some FCC auctions (see Ayres and Cramton, 1996). We will come back to the preferential treatment issue when we discuss implementing asymmetric auctions in Section 3.

Our sufficient condition for the asymmetry of the optimal auction depends on both the magnitude of the participation cost and the number of bidders through the optimal symmetric cutoff $v^{s}$. For certain distribution functions (for example, uniform with $v_{h}<2 v_{l}$ ) this sufficient condition will always be

\footnotetext{
${ }^{38}$ Let $\widetilde{v}_{1}<v^{s}<\widetilde{v}_{2}$, where $2 F\left(v^{s}\right)=F\left(\widetilde{v}_{1}\right)+F\left(\widetilde{v}_{2}\right)$. The probability of making a sale is $1-F\left(v^{s}\right)^{2}$ when the cutoffs are symmetric, and $1-F\left(\widetilde{v}_{1}\right) F\left(\widetilde{v}_{2}\right)$ when they are asymmetric. The latter is larger than the former, since $F\left(v^{s}\right)^{2}-F\left(\widetilde{v}_{1}\right) F\left(\widetilde{v}_{2}\right)=F\left(v^{s}\right)^{2}-F\left(\widetilde{v}_{1}\right)\left(2 F\left(v^{s}\right)-\right.$ $\left.F\left(\widetilde{v}_{1}\right)\right)=\left(F\left(v^{s}\right)-F\left(\widetilde{v}_{1}\right)\right)^{2}>0$.

${ }^{39}$ This is the case unless $v_{0}=v_{l}$ (with $J\left(v_{l}\right)>0$ ) making it impossible to even create the type of asymmetry we are considering.

${ }^{40} \mathrm{We}$ are considering the regular case of increasing virtual valuation functions.
} 
The B.E. Journal of Theoretical Economics, Vol. 9 [2009], Iss. 1 (Advances), Art. 24

satisfied, i.e., the optimal auction will be asymmetric regardless of the participation cost level and the number of bidders. ${ }^{41}$

Corollary 1 If $\frac{J(v)}{F(v)}$ is decreasing on $\left(v_{l}, v_{h}\right)$, then the optimal auction is asymmetric (independent of $c$ and $n$ ).

The optimal auction is symmetric when $c=0$, with the cutoff $v_{0}$. The corollary identifies cases where even an infinitesimally small $c$ causes the optimal auction to be asymmetric. However, the asymmetry will also be small: As $c$ approaches to 0, bidders' optimal cutoffs all approach to $v_{0}$. In other words, even though there is no "continuity" in the symmetry property of the optimal auction at $c=0$, there is continuity in terms of outcomes, and hence the seller's profit.

We next turn our attention to conditions under which the optimal auction is symmetric.

Proposition 2 (SYMMETRY) The optimal auction is symmetric for all $c$ (and n), i.e., $\widetilde{v}_{i}^{*}=v^{s} \forall i \in N$, if and only if $\frac{J(v)}{F(v)}$ is weakly increasing on $\left(v_{0}, v_{h}\right)$.

The necessity part of the result is a consequence of Proposition 1. If $\frac{J(v)}{F(v)}$ is not weakly increasing at some $v^{\prime}$ in $\left(v_{0}, v_{h}\right)$, then, for any given number of bidders, we can find a participation cost level for which the optimal symmetric cutoff $v^{s}$ equals to $v^{\prime}$, so that the sufficient condition of Proposition 1 is satisfied, i.e., the optimal auction is asymmetric. ${ }^{42}$

The main interest in Proposition 2 stems from the sufficiency part. If the distribution of valuations is such that $\frac{J(v)}{F(v)}$ is weakly increasing on the relevant range, then the optimal auction is symmetric and hence completely characterized: Each bidder has the same participation cutoff $v^{s}$, as defined in (10). For this result, obviously, it is not enough to consider only local improvements around $v^{s}$. In order to gain some understanding for the result and the condition, consider the two bidders case with asymmetric cutoffs, i.e., $\widetilde{v}_{1}<\widetilde{v}_{2}$. Suppose the seller increases $\widetilde{v}_{1}$ and decreases $\widetilde{v}_{2}$ slightly in such a way that total participation cost incurred stays the same. As a result of these changes in the cutoffs, the seller's profit from bidder 1 (net of the participation

\footnotetext{
${ }^{41}$ Since $v_{0}<v^{s}<v_{h}$, we need $\frac{J(v)}{F(v)}$ to be decreasing only on $\left(v_{0}, v_{h}\right)$ for this result. However, when $v_{0}>v_{l}, \frac{J(v)}{F(v)}$ cannot be decreasing on $\left(v_{0}, v_{h}\right)$ (since $\frac{J\left(v_{0}\right)}{F\left(v_{0}\right)}=0$ and $\frac{J\left(v_{h}\right)}{F\left(v_{h}\right)}=$ $\left.v_{h}\right)$, so this case is irrelevant.

${ }^{42}$ We can see from the definition of $v^{s}$ in (10) that $v^{s}$ is a continuous and increasing function of $c$ (for any given $n$ ), where $v^{s} \rightarrow v_{0}$ as $c \rightarrow 0$ and $v^{s} \rightarrow v_{h}$ as $c \rightarrow v_{h}$.
} 
Celik and Yilankaya: Optimal Auctions with Simultaneous and Costly Participation

cost) decreases by $J\left(\widetilde{v}_{1}\right) F\left(\widetilde{v}_{2}\right)+\int_{\widetilde{v}_{1}}^{\widetilde{v}_{2}} J(v) f(v) d v$, where the first term arises from increasing $\widetilde{v}_{1}$ slightly and the second term is the result of types in $\left(\widetilde{v}_{1}, \widetilde{v}_{2}\right)$ receiving the object with a lower probability due to a decrease in $\widetilde{v}_{2}$. This loss is bounded above by $J\left(\widetilde{v}_{1}\right) F\left(\widetilde{v}_{2}\right)+J\left(\widetilde{v}_{2}\right)\left[F\left(\widetilde{v}_{2}\right)-F\left(\widetilde{v}_{1}\right)\right]$. On the other hand, the profit from bidder 2 (again, net of the participation cost) increases by $J\left(\widetilde{v}_{2}\right) F\left(\widetilde{v}_{2}\right)$ due to the decrease in $\widetilde{v}_{2}$. Therefore, the seller's profit will increase if $J\left(\widetilde{v}_{2}\right) F\left(\widetilde{v}_{1}\right)-J\left(\widetilde{v}_{1}\right) F\left(\widetilde{v}_{2}\right) \geq 0$, or $\frac{J\left(\widetilde{v}_{2}\right)}{F\left(\widetilde{v}_{2}\right)} \geq \frac{J\left(\widetilde{v}_{1}\right)}{F\left(\widetilde{v}_{1}\right)}$.

Remark 2 For distribution functions that satisfy the monotone hazard rate condition $\left(\frac{1-F(v)}{f(v)}\right.$ is decreasing), if $\frac{v}{F(v)}$ is increasing, then so is $\frac{J(v)}{F(v)}$. Therefore, if $v_{l}=0, F(v)$ is concave and satisfies the monotone hazard rate condition, then the optimal auction will be symmetric.

We next present two results about the nature of (possible) asymmetries in the optimal auction. First, we show that, when the sufficient condition for the asymmetry of the optimal auction in Corollary 1 is satisfied, only one bidder will have the lowest cutoff. Second, we identify a class of distribution functions for which the optimal auction is either symmetric or uses only two cutoffs. Notice that both of these results are independent of the number of bidders and the magnitude of the participation cost, and they simplify the task of finding the optimal auction considerably whenever they apply.

Proposition 3 i) If $\frac{J(v)}{F(v)}$ is decreasing on $\left(v_{l}, v_{h}\right)$, then in the optimal auction only one bidder has the lowest cutoff, i.e., $\widetilde{v}_{i}^{*}>\widetilde{v}_{1}^{*}$ for all $i \in\{2, \ldots, n\}$.

ii) If $J^{\prime}(v) \frac{F(v)}{f(v)}$ is weakly increasing on $\left(v_{0}, v_{h}\right)$, then the optimal auction has at most two distinct cutoffs.

The proof of part (i) parallels that of Proposition 1: If $\frac{J(v)}{F(v)}$ is decreasing and two or more bidders are using the lowest cutoff, the seller can increase her profit by splitting these bidders into two arbitrary groups and setting a different cutoff for each group. In a similar fashion, we can find conditions for optimality of separating or bunching different bidders who are using cutoffs other than the lowest one. There is one additional complication: Changing the lowest cutoff affects the seller's rent extraction only from bidders using that cutoff, since it does not alter the expected probability of winning for the other bidders. However, changing a higher cutoff also affects the seller's revenue from bidders using lower cutoffs than the modified one. Therefore, conditions regarding bunching bidders at higher cutoffs are different from conditions for bunching them at the lowest cutoff: Having $J^{\prime}(v) \frac{F(v)}{f(v)}$ weakly increasing is sufficient to rule out the optimality of separating bidders into three or more groups with different cutoffs. 
The B.E. Journal of Theoretical Economics, Vol. 9 [2009], Iss. 1 (Advances), Art. 24

\subsection{Uniform Distributions}

In this section, using our previous results, we characterize optimal auctions when bidders' valuations are uniformly distributed and provide some comparative statics results.

We have $n \geq 2$ bidders whose valuations are uniformly distributed on $\left[v_{l}, v_{h}\right]$, where $0 \leq v_{l}<v_{h}$, i.e., $F(v)=\frac{v-v_{l}}{v_{h}-v_{l}}$. The participation cost is $c \in\left(0, v_{h}\right)$. The virtual valuation function is given by $J(v)=2 v-v_{h}$, which is increasing. If $2 v_{l}-v_{h} \geq 0$, then $v_{0}=v_{l}$; otherwise $v_{0}=\frac{v_{h}}{2}$. When $c=0$, the object is assigned to the highest valuation bidder in the optimal auction as long as her valuation is higher than $v_{0}$.

We first observe that $J^{\prime}(v) \frac{F(v)}{f(v)}=2\left(v-v_{l}\right)$ is increasing. Therefore, at most two distinct cutoffs will be used in the optimal auction (Proposition 3ii). We next note that $\frac{J(v)}{F(v)}=\frac{\left(2 v-v_{h}\right)\left(v_{h}-v_{l}\right)}{v-v_{l}}$ is either weakly increasing (if $v_{h} \geq 2 v_{l}$ ) or decreasing (if $v_{h}<2 v_{l}$ ) on the entire support $\left[v_{l}, v_{h}\right]$. So, if $v_{h} \geq 2 v_{l}$, then it follows from Proposition 2 that the optimal auction is symmetric. The optimal cutoffs are given by $\widetilde{v}_{1}^{*}=\ldots=\widetilde{v}_{n}^{*}=v^{s}$, where

$$
J\left(v^{s}\right) F\left(v^{s}\right)^{n-1}=\left(2 v^{s}-v_{h}\right)\left(\frac{v^{s}-v_{l}}{v_{h}-v_{l}}\right)^{n-1}=c .
$$

If $v_{h}<2 v_{l}$, then the optimal auction is asymmetric (Corollary 1 ) with exactly two cutoffs. Moreover, only one bidder will have the lower cutoff (Proposition 3i). Using these, solving the seller's problem becomes a straightforward exercise. We provide the solution here for completeness. Let $\widetilde{v}_{1}^{*}=a$ and $\widetilde{v}_{2}^{*}=\ldots=\widetilde{v}_{n}^{*}=b>a$.

- If $c \leq \min \left\{v_{h}-v_{l}, \frac{\left(2 v_{l}-v_{h}\right)^{n}}{\left(v_{h}-v_{l}\right)^{n-1}}\right\}$, then $a=v_{l}$ and $b=v_{l}+c^{\frac{1}{n}}\left(v_{h}-v_{l}\right)^{\frac{n-1}{n}}$.

- If $v_{h}-v_{l}<c<2 v_{l}-v_{h}$, then $a=v_{l}$ and $b=v_{h}$.

- If $\frac{\left(2 v_{l}-v_{h}\right)^{n}}{\left(v_{h}-v_{l}\right)^{n-1}}<c<3 v_{h}-4 v_{l}$, then $a$ satisfies $\left(2 a-v_{h}\right)\left(\frac{a+v_{l}-v_{h}}{v_{h}-v_{l}}\right)^{n-1}=c$ and $b=a+2 v_{l}-v_{h}$.

- If $c \geq \max \left\{2 v_{l}-v_{h}, 3 v_{h}-4 v_{l}\right\}$, then $a=\frac{v_{h}+c}{2}$ and $b=v_{h} \cdot{ }^{43}$

Note that the optimal cutoffs are weakly increasing in $n$. If $v_{h} \geq 2 v_{l}$, then the optimal auction is symmetric, and as $n$ increases the seller chooses to restrict participation symmetrically, i.e., $v^{s}$ is increasing in $n$ with $v^{s} \rightarrow v_{h}$

\footnotetext{
${ }^{43}$ Note that when $v_{h}<2 v_{l}$ we have, $v_{h}-v_{l}<\frac{\left(2 v_{l}-v_{h}\right)^{n}}{\left(v_{h}-v_{l}\right)^{n-1}} \Leftrightarrow v_{h}-v_{l}<2 v_{l}-v_{h} \Leftrightarrow$ $\frac{\left(2 v_{l}-v_{h}\right)^{n}}{\left(v_{h}-v_{l}\right)^{n-1}}>3 v_{h}-4 v_{l} \Leftrightarrow 2 v_{l}-v_{h}>3 v_{h}-4 v_{l}$.
} 
Celik and Yilankaya: Optimal Auctions with Simultaneous and Costly Participation

as $n \rightarrow \infty$. If $v_{h}<2 v_{l}$, both $a$ and $b$ are weakly increasing in $n$, and $b \rightarrow v_{h}$ as $n \rightarrow \infty$.

The optimal cutoffs are also weakly increasing in $c$. All cutoffs approach $v_{0}$ as $c \rightarrow 0$ and $v_{h}$ as $c \rightarrow v_{h}$.

Dealing exclusively with one bidder, or "sole-sourcing," is a commonly observed phenomenon in government procurement. In our setting, sole source contracting emerges as an optimal response to high participation costs in certain cases: Whenever the optimal auction is asymmetric, the seller deals with one of the bidders exclusively when the participation cost is high enough. Observe that this threshold cost level, and hence whether sole-sourcing is optimal or not, is independent of the number of bidders.

\section{Implementing the Optimal Auction}

Our objective in this section is to show that using common auction formats, augmented with "familiar" instruments or variations, can be optimal for the seller. ${ }^{44}$ This task is trivial if the optimal auction is symmetric, i.e., each bidder has the same cutoff $v^{s}$, defined in (10). The standard auctions, e.g., first and second price auctions (FPA and SPA, respectively), with appropriately chosen reserve price and/or entry fee will be optimal. To see this, let $r$ denote the reserve price and $c^{e}$ effective participation cost, i.e., $c^{e}$ is the sum of the participation cost $c$ and the entry fee (which could be negative, implying an entry subsidy). Suppose $r$ and $c^{e}$ satisfy the following equation:

$$
\left(v^{s}-r\right) F\left(v^{s}\right)^{n-1}=c^{e} .
$$

Both FPA and SPA, with $r$ and $c^{e}$ satisfying (11), are optimal, since each has a symmetric equilibrium where all bidders use the participation cutoff $v^{s}$ (at which their expected payoffs are zero) and their bids are increasing in their valuations, implying that the highest-valuation participant receives the object.

Our results concerning second price auctions are valid also for English auctions as long as bidders make the (costly) participation decision prior to the start of bid calling, which is assumed to be costless: Participating bidders will bid their valuations in all (undominated) equilibria regardless of what they observe about the set of participants.

\footnotetext{
${ }^{44}$ We will not be concerned with "strong implementation" in what follows. So, we call an auction form optimal if the seller obtains her maximal profit in one (as opposed to all) of its (Bayesian-Nash) equilibria.
} 
The seller can accomplish her goal in a simple way that also works when the optimal auction is asymmetric. Consider a SPA where each bidder has an individualized reserve price given by her optimal cutoff (only bids exceeding her reserve price are allowable), and an entry subsidy of $c$ is provided to any bidder who submits an allowable bid, i.e., the effective participation cost is zero. There is an equilibrium in dominant strategies where bidders participate (and bid their valuations) iff their valuations are greater than their respective reserve prices. This gives the seller her maximal profit, since the object is assigned to the highest-valuation participant and bidders use the optimal cutoffs where their expected payoffs are zero.

We nevertheless believe that further investigation of implementing asymmetric optimal cutoffs is a worthwhile endeavor for two reasons. First, the above auction is not anonymous, i.e., the bidders are not treated identically by its rules. Second, even when non-anonymous auctions are used in practice (we provide a few examples below), bidder-specific reserve prices have never been employed, to the best of our knowledge. In what follows, we will first show that under some conditions the seller can obtain her maximal profit by using an anonymous auction. We will then discuss some non-anonymous auctions that resemble the ones that are actually observed in practice.

\subsection{Anonymous Second Price Auctions}

There may be multiple equilibria (in undominated strategies) in SPAs with costly participation even in the symmetric independent private values environment we are considering. ${ }^{45}$ In any equilibrium in undominated strategies, bidders employ cutoff rules in participation and bid their valuations whenever they submit a bid. There is always a symmetric equilibrium where the cutoffs used are all identical, but there may be asymmetric equilibria as well. Therefore, it may be possible for the seller to achieve her optimal profit level in an asymmetric equilibrium of an anonymous SPA. To demonstrate this point, we return to Example 1, where there are two bidders, $F(v)=v^{4}$ on $[0,1]$, and $c=0.4$. The optimal auction is asymmetric, with $\widetilde{v}_{1}^{*} \approx .816$ and $\widetilde{v}_{2}^{*} \approx .92$. Now, consider a SPA with reserve price $r \approx .598$ and effective participation $\operatorname{cost} c^{e} \approx .156$ (there is an entry subsidy). There is an equilibrium where one of the bidders participate iff her valuation is greater than .816, the other use .92 as her cutoff, and both bid their valuations whenever they participate. ${ }^{46}$ In

\footnotetext{
${ }^{45}$ See Campbell (1998) and Tan and Yilankaya (2006) for conditions under which this would happen.

${ }^{46}$ There is also a symmetric equilibrium which gives the seller a lower profit.
} 
this equilibrium, the highest-valuation participant receives the object. In addition, the expected payoffs of bidders are zero at their respective cutoffs, since these are determined by indifference (to participation) conditions. Therefore, the seller obtains her optimal profit.

This example can be generalized as follows:

Proposition 4 Suppose that the optimal auction has two cutoffs, and that the monotone hazard rate condition is satisfied $\left(\frac{1-F(v)}{f(v)}\right.$ is decreasing). An anonymous second price auction (with appropriately chosen reserve price and effective participation cost) has an equilibrium in undominated strategies that is optimal for the seller.

Two cutoff requirement is obviously an important restriction. ${ }^{47}$ However, we know that under some conditions the optimal auction will indeed have at most two distinct cutoffs (Proposition 3ii provides a sufficient condition). Moreover, whenever our sufficient condition for the asymmetry of the optimal auction is satisfied, the seller needs to implement only two distinct cutoffs to improve over the optimal symmetric cutoff $v^{s}$ (Proposition 1), which can again be accomplished by using an anonymous SPA. ${ }^{48}$

We use the optimality of the cutoffs and the monotone hazard rate condition to make sure that the reserve price is nonnegative. If we do not impose this restriction (a negative reserve price implies that a bidder gets a subsidy if she is the only participant), then no assumptions are needed: Virtually all arbitrary pairs of cutoffs are equilibrium cutoffs for some anonymous second price auction. ${ }^{49}$

\subsection{Differential Effective Participation Costs}

Not all bidders incur the same participation cost in all auctions, and sometimes this happens by the design of the seller. One obvious way of doing this is by charging bidders different entry fees. There are also indirect ways. The seller may provide guaranteed financing for some bidders, thus saving them the fixed costs associated with credit arrangements. This was done, for example, in the FCC spectrum auctions; see, e.g., Ayres and Cramton (1996). Also, the rules

\footnotetext{
${ }^{47}$ For example, when there are three bidders, $F(v)=v^{4}$ on $[0,1]$, and $c=0.327$, the optimal auction has three distinct cutoffs and cannot be implemented using an anonymous SPA with any $r$ and $c^{e}$ pair.

${ }^{48}$ The proof of this result is contained in our proof of Proposition 4 in the Appendix.

${ }^{49}$ The only exception is if the lower cutoff is $v_{l}$ and two or more bidders are supposed to use it. Note that this will never be optimal. (See the proof of Proposition 4 in the Appendix.)
} 
The B.E. Journal of Theoretical Economics, Vol. 9 [2009], Iss. 1 (Advances), Art. 24

of the auction may be such that some bidders face higher participation costs. For example, participation costs of foreign firms are sometimes increased in government procurement by imposing residency requirements, giving a very tight deadline for submission of bids, etc.; see, e.g., McAfee and McMillan (1989).

If the seller can induce differential effective participation costs, then a SPA or FPA will be optimal for the seller. We demonstrate these for the two-bidders case for expositional simplicity. Let $\widetilde{v}_{1}^{*}$ be the cutoff of bidder 1 and $\widetilde{v}_{2}^{*}>\widetilde{v}_{1}^{*}$ that of bidder 2 in the optimal auction. Consider the SPA with $r=\widetilde{v}_{1}^{*}, c_{1}^{e}=0$, and $c_{2}^{e}=\int_{\widetilde{v}_{1}^{*}}^{\widetilde{v}_{2}^{*}} F(v) d v$, where $c_{i}^{e}$ is the effective participation cost of bidder $i$. It is a dominant strategy for the first bidder to participate and bid her valuation iff her valuation is greater than $\widetilde{v}_{1}^{*}$. Given this, the second bidder's expected payoff (for $v_{2}>\widetilde{v}_{1}^{*}$ ) if she participates and bids her valuation is

$$
\left(v_{2}-\widetilde{v}_{1}^{*}\right) F\left(\widetilde{v}_{1}^{*}\right)+\int_{\widetilde{v}_{1}^{*}}^{v_{2}}\left(v_{2}-v\right) d F(v)-c_{2}^{e}=\int_{\widetilde{v}_{1}^{*}}^{v_{2}} F(v) d v-c_{2}^{e} .
$$

Note that $c_{2}^{e}$ is chosen in such a way that bidder 2 participates (and bids her valuation) iff her valuation is greater than $\widetilde{v}_{2}^{*}$. Therefore, the seller obtains her maximal profit. ${ }^{50}$

The seller can also achieve her goal by using the FPA with $r=\widetilde{v}_{1}^{*}, c_{1}^{e}=0$, and $c_{2}^{e}=\int_{\widetilde{v}_{1}^{*}}^{\widetilde{v}_{2}^{*}} F\left(\widetilde{v}_{2}^{*}\right) d v$, since there is an equilibrium of this auction where $i$ uses $\widetilde{v}_{i}^{*}$ as her cutoff (at which her expected payoff is zero) and both bidders use the same strictly increasing bid function for types greater than $\widetilde{v}_{2}^{*}$, so that the highest-valuation participant receives the object. ${ }^{51}$ To calculate the bid functions, and to see where these effective participation costs are coming from, suppose such an equilibrium exists. ${ }^{52}$ Let $Q_{i}^{*}(\cdot)$ be $i$ 's probability of winning function in this equilibrium (and hence in the optimal auction). From the

\footnotetext{
${ }^{50}$ For arbitrary $n$, the same method would yield the SPA with $r=\widetilde{v}_{1}^{*}$ and $c_{i}^{e}=$ $\sum_{j=1}^{i-1} \prod_{\substack{k=j+1 \\ k \neq i}}^{n+1} F\left(\widetilde{v}_{k}^{*}\right) \int_{\widetilde{v}_{j}^{*}}^{\widetilde{v}_{j+1}^{*}} F(v)^{j} d v, \forall i \in N$.

${ }^{51}$ The equilibrium bid functions for arbitrary $n$ are given by (14) as well, so the FPA with $r=\widetilde{v}_{1}^{*}$ and $c_{i}^{e}=\int_{\widetilde{v}_{1}^{*}}^{\widetilde{v}_{i}^{*}} Q_{1}^{*}(v) d v=\sum_{j=1}^{i-1} \prod_{k=j+1}^{n+1} F\left(\widetilde{v}_{k}^{*}\right) \int_{\widetilde{v}_{j}^{*}}^{\widetilde{v}_{j+1}^{*}} F(v)^{j-1} d v, \forall i \in N$, will be optimal.

${ }^{52}$ The bid functions we find below indeed constitute an equilibrium. The proof is identical to that of the similar claim for standard FPAs.
} 
incentive compatibility conditions, we have, for $v \geq \widetilde{v}_{i}^{*}$,

$$
\pi_{i}(v)=Q_{i}^{*}(v) v-P_{i}(v)=\int_{\widetilde{v}_{i}^{*}}^{v} Q_{i}^{*}(y) d y,
$$

where

$$
P_{i}(v)=c_{i}^{e}+b_{i}(v) Q_{i}^{*}(v)
$$

is $i$ 's equilibrium expected payment and $b_{i}(\cdot)$ is $i$ 's equilibrium bid. Combining (12) and (13),

$$
b_{i}(v)=v-\frac{\int_{\widetilde{v}_{i}^{*}}^{v} Q_{i}^{*}(y) d y+c_{i}^{e}}{Q_{i}^{*}(v)} .
$$

Notice that $b_{i}^{\prime}(v)>0$. Consider $v>\widetilde{v}_{2}^{*}$. We have $Q_{1}^{*}(v)=Q_{2}^{*}(v)=F(v)$, since both participate and the highest-valuation participant wins, and so $b_{1}(v)=$ $b_{2}(v)$ if $c_{1}^{e}=0$ and $c_{2}^{e}=\int_{\widetilde{v}_{1}^{*}}^{\widetilde{v}_{2}^{*}} Q_{1}^{*}(y) d y=\int_{\widetilde{v}_{1}^{*}}^{\widetilde{v}_{*}^{*}} F\left(\widetilde{v}_{2}^{*}\right) d v$.

\subsection{Bidding Preferences}

In some government auctions certain groups of bidders are given explicit bidding preferences. For example, the Buy American Act of the US (and comparable provisions in other countries) gives bidding preferences to domestic firms over foreign firms in government procurement. Similarly, small businesses or in-state bidders are favored in some government auctions.

We now show that, in our setup, a FPA with bidding preferences could be optimal for the seller. To see this, first note that in the optimal auction, bidder $i$ 's expected payment is given by, see (12) for example,

$$
P_{i}^{*}(v)=Q_{i}^{*}(v) v-\int_{\widetilde{v}_{i}^{*}}^{v} Q_{i}^{*}(y) d y,
$$

where $Q_{i}^{*}(\cdot)$ is $i$ 's probability of winning function (given by (8) and the optimal cutoffs). Now consider the FPA with $r=\widetilde{v}_{1}^{*}$ and effective bid functions, for all $i \in N$,

$$
\delta_{i}(b)=\left\{\begin{array}{cc}
\widetilde{v}_{i}^{*}-\frac{c}{Q_{i}^{*}\left(v_{i}^{*}\right)} & \widetilde{v}_{1}^{*} \leq b<\widetilde{v}_{i}^{*} \\
b-\frac{\int_{\widetilde{v}_{i}^{*}}^{b} Q_{i}^{*}(v) d v+c}{Q_{i}^{*}(b)} & \widetilde{v}_{i}^{*} \leq b \leq v_{h} \\
b-\left(\int_{\widetilde{v}_{i}^{*}}^{v_{h}} Q_{i}^{*}(v) d v+c\right) & v_{h}<b
\end{array}\right.
$$

so that bidder $i$ receives the object if her bid $b$ is the highest bid (as long as it is higher than the reserve price $\widetilde{v}_{1}^{*}$ ), but pays only her effective bid $\delta_{i}(b)$ rather than her actual bid $b$. There is an equilibrium of this auction where 
The B.E. Journal of Theoretical Economics, Vol. 9 [2009], Iss. 1 (Advances), Art. 24

bidder $i$ participates iff her valuation is higher than $\widetilde{v}_{i}^{*}$ and all participating bidders bid their valuations, giving the seller her maximal profit. To see that this is indeed an equilibrium, suppose that all bidders but $i$ are following their equilibrium strategies. Bidding $\widetilde{v}_{i}^{*}$ is better than bidding anything lower, since the winning probability is higher (strictly, unless $i=1$ ) and the effective bid, i.e., the payment conditional on winning, is the same. Similarly, bidding $v_{h}$ is better than bidding anything higher, since the winning probability is constant and the effective bid is lower. Finally, note that $i$ 's effective bidding function is constructed so that if she bids $v^{\prime} \in\left[\widetilde{v}_{i}^{*}, v_{h}\right]$, then her expected probability of winning is $Q_{i}^{*}\left(v^{\prime}\right)$ and her expected payment is $P_{i}^{*}\left(v^{\prime}\right)$, i.e., we have $\delta_{i}\left(v^{\prime}\right) Q_{i}^{*}\left(v^{\prime}\right)+c=P_{i}^{*}\left(v^{\prime}\right)$. Since the optimal auction is incentive compatible and individually rational, it is a best-response for $i$ to participate (and bid her valuation) iff her valuation is higher than $\widetilde{v}_{i}^{*}$.

\section{Appendix}

Proof of Proposition 1. Fix an integer $k$ such that $1 \leq k<n$. Suppose that the seller considers only two cutoff auctions, where the cutoff of the first $k$ bidders is $a$ and the others' is $b \geq a$. The expected profit of the seller in terms of $a$ and $b$ is

$$
\begin{aligned}
R(a, b)= & \int_{a}^{b} J(v) F(b)^{n-k} d F(v)^{k}+\int_{b}^{v_{h}} J(v) d F(v)^{n} \\
& -k c(1-F(a))-(n-k) c(1-F(b)) .
\end{aligned}
$$

$R_{a a}, R_{b b}<0$ at $v^{s}$, using the standard notation for second derivatives. At $a=b=v^{s}$, using the fact that $R_{a}=R_{b}=0$,

$$
\begin{gathered}
\frac{R_{a a}}{R_{a b}}=\frac{J^{\prime}\left(v^{s}\right) F\left(v^{s}\right)+(k-1) J\left(v^{s}\right) f\left(v^{s}\right)}{(n-k) J\left(v^{s}\right) f\left(v^{s}\right)}>0, \\
\frac{R_{a b}}{R_{b b}}=\frac{k J\left(v^{s}\right) f\left(v^{s}\right)}{J^{\prime}\left(v^{s}\right) F\left(v^{s}\right)+(n-k-1) J\left(v^{s}\right) f\left(v^{s}\right)}>0 .
\end{gathered}
$$

Therefore, if $\frac{J(v)}{F(v)}$ is strictly decreasing at $v^{s}$, then

$$
0<\frac{R_{a a}}{R_{a b}}<\frac{R_{a b}}{R_{b b}}
$$

at $a=b=v^{s}$, proving the result: We can find $\epsilon_{1}, \epsilon_{2}>0$ such that $R\left(v^{s}-\right.$ 
Celik and Yilankaya: Optimal Auctions with Simultaneous and Costly Participation

$\left.\epsilon_{1}, v^{s}+\epsilon_{2}\right)>R\left(v^{s}, v^{s}\right) .{ }^{53}$

Proof of Proposition 2. The necessity part is straightforward and discussed in the text. For sufficiency, suppose to the contrary that $\frac{J(v)}{F(v)}$ is weakly increasing on $\left(v_{0}, v_{h}\right)$, but the optimal auction is asymmetric, so that at least two distinct cutoffs are chosen. Consider two smallest cutoffs: $a \geq v_{0}$ is used for bidders $1, \ldots, m$, and $b>a$ is used for bidders $m+1, \ldots, m^{\prime}$, where $1 \leq m<m^{\prime} \leq n$. From the first order condition for $a$,

$$
c-J(a) F(a)^{m-1} F(b)^{m^{\prime}-m} \prod_{k=m^{\prime}+1}^{n+1} F\left(\widetilde{v}_{k}^{*}\right) \leq 0,
$$

which is satisfied with equality whenever $a>v_{l}$.

From the first order condition with respect to $b$,

$c-J(b) F(b)^{m^{\prime}-1} \prod_{k=m^{\prime}+1}^{n+1} F\left(\widetilde{v}_{k}^{*}\right)+F(b)^{m^{\prime}-m-1} \prod_{k=m^{\prime}+1}^{n+1} F\left(\widetilde{v}_{k}^{*}\right) \int_{a}^{b} J(v) d F(v)^{m} \geq 0$,

which is satisfied with equality whenever $b<v_{h}$. Combining these, we have

$$
\begin{aligned}
J(a) F(a)^{m-1} F(b) & \geq J(b) F(b)^{m}-\int_{a}^{b} J(v) d F(v)^{m} \\
& >J(b) F(b)^{m}-J(b)\left(F(b)^{m}-F(a)^{m}\right) \\
& =J(b) F(a)^{m}
\end{aligned}
$$

or,

$$
\frac{J(a)}{F(a)}>\frac{J(b)}{F(b)}
$$

which is a contradiction.

Proof of Proposition 3. i) Suppose by contradiction that $\widetilde{v}_{1}^{*}$ is the cutoff of the first $m>1$ bidders in the optimal auction. Let $k$ be an arbitrary positive integer smaller than $m$. Consider the class of auctions, where the first $k$ cutoffs are equal to $a$, the following $m-k$ cutoffs are equal to $b$, and cutoffs $m+1$ to $n$ are given as $\widetilde{v}_{m+1}^{*}$ to $\widetilde{v}_{n}^{*}$, such that $a<b<\widetilde{v}_{m+1}^{*}$. We can write the

\footnotetext{
${ }^{53} \mathrm{At}\left(v^{s}, v^{s}\right)$ we are on the boundary of the feasible set ( $b \geq a$ constraint), so showing that the Hessian is not negative definite at $\left(v^{s}, v^{s}\right)$ would not have been sufficient. The inequalities in (15), which imply that the Hessian is not negative definite, but not implied by it, ensure that there is an improvement by "moving towards the feasible side of the boundary."
} 
The B.E. Journal of Theoretical Economics, Vol. 9 [2009], Iss. 1 (Advances), Art. 24

expected profit from such an auction as a function of $a$ and $b$ :

$$
\begin{aligned}
R(a, b)= & k \int_{a}^{b} J(v)\left[F(v)^{k-1} F(b)^{m-k} \prod_{j=m+1}^{n+1} F\left(\widetilde{v}_{j}^{*}\right)\right] f(v) d v \\
& +m \int_{b}^{\widetilde{v}_{m+1}^{*}} J(v)\left[F(v)^{m-1} \prod_{j=m+1}^{n+1} F\left(\widetilde{v}_{j}^{*}\right)\right] f(v) d v \\
& -k c(1-F(a))-(m-k) c(1-F(b)) \\
& +\sum_{i=m+1}^{n} i \int_{\widetilde{v}_{i}^{*}}^{\widetilde{v}_{i+1}^{*}} J(v)\left[F(v)^{i-1} \prod_{j=i+1}^{n+1} F\left(\widetilde{v}_{j}^{*}\right)\right] f(v) d v \\
& -c \sum_{i=m+1}^{n}\left(1-F\left(\widetilde{v}_{i}^{*}\right)\right) .
\end{aligned}
$$

The optimal auction must also be optimal within this class. Therefore, $R(a, b)$ is maximized at $a=b=\widetilde{v}_{1}^{*}$. First, note that, since $\frac{J(v)}{F(v)}$ is decreasing on $\left[v_{l}, v_{h}\right]$, the optimal auction is asymmetric (Corollary 1 ), i.e., $\widetilde{v}_{1}^{*}<v_{h}$. Note also that $\widetilde{v}_{1}^{*}>v_{l}$, since when $a=b=v_{l}$, the first order condition for $a$ is violated, i.e.,

$$
R_{a}\left(v_{l}, v_{l}\right)=k f\left(v_{l}\right)\left[c-J\left(v_{l}\right) F\left(v_{l}\right)^{m-1} \prod_{j=m+1}^{n+1} F\left(\widetilde{v}_{j}^{*}\right)\right]>0,
$$

since $F\left(v_{l}\right)=0$ and $f\left(v_{l}\right)>0$. Hence, $a=b=\widetilde{v}_{1}^{*}$ could satisfy the first order necessary conditions only at an interior point. Following the proof of Proposition 1, note that, at $a=b=\widetilde{v}_{1}^{*}$, we have

$$
\begin{gathered}
\frac{R_{a a}}{R_{a b}}=\frac{J^{\prime}\left(v_{1}^{*}\right) F\left(v_{1}^{*}\right)+(k-1) J\left(v_{1}^{*}\right) f\left(v_{1}^{*}\right)}{(m-k) J\left(v_{1}^{*}\right) f\left(v_{1}^{*}\right)}>0, \\
\frac{R_{a b}}{R_{b b}}=\frac{k J\left(v_{1}^{*}\right) f\left(v_{1}^{*}\right)}{J^{\prime}\left(v_{1}^{*}\right) F\left(v_{1}^{*}\right)+(m-k-1) J\left(v_{1}^{*}\right) f\left(v_{1}^{*}\right)}>0 .
\end{gathered}
$$

Therefore, if $J^{\prime}\left(\widetilde{v}_{1}^{*}\right) F\left(\widetilde{v}_{1}^{*}\right)<J\left(\widetilde{v}_{1}^{*}\right) f\left(\widetilde{v}_{1}^{*}\right)$, i.e., $\frac{J(v)}{F(v)}$ is decreasing at $\widetilde{v}_{1}^{*}$, then $\frac{R_{a a}}{R_{a b}}<\frac{R_{a b}}{R_{b b}}$ at $a=b=\widetilde{v}_{1}^{*}$, implying that $a=b=\widetilde{v}_{1}^{*}$ cannot be optimal, a contradiction.

ii) The proof is by contradiction. Suppose to the contrary that at least three cutoffs are used in the optimal auction, and consider three smallest of these cutoffs, $v_{l} \leq a_{1}<a_{2}<a_{3} \leq v_{h}$, where the number of bidders using these cutoffs are $n_{1}, n_{2}$, and $n_{3}$ respectively. From the first order condition 
Celik and Yilankaya: Optimal Auctions with Simultaneous and Costly Participation

with respect to the cutoffs of $n_{1}$ bidders who use $a_{1} \geq v_{0}$ (using (9)), we have

$$
c-J\left(a_{1}\right) F\left(a_{1}\right)^{n_{1}-1} F\left(a_{2}\right)^{n_{2}} F\left(a_{3}\right)^{n_{3}} \prod_{j=n_{1}+n_{2}+n_{3}+1}^{n+1} F\left(\widetilde{v}_{j}^{*}\right) \leq 0
$$

with equality if $a_{1}>v_{l}$.

From the first order condition with respect to the cutoffs of bidders using $a_{2}$

$$
\begin{aligned}
c-\left[J\left(a_{2}\right) F\left(a_{2}\right)^{n_{1}}-n_{1} \int_{a_{1}}^{a_{2}} J(v) F(v)^{n_{1}-1} f(v) d v\right] \\
F\left(a_{2}\right)^{n_{2}-1} F\left(a_{3}\right)^{n_{3}} \prod_{j=n_{1}+n_{2}+n_{3}+1}^{n+1} F\left(\widetilde{v}_{j}^{*}\right)=0,
\end{aligned}
$$

or, after integration by parts,

$c=\left[J\left(a_{1}\right) F\left(a_{1}\right)^{n_{1}}+\int_{a_{1}}^{a_{2}} J^{\prime}(v) F(v)^{n_{1}} d v\right] F\left(a_{2}\right)^{n_{2}-1} F\left(a_{3}\right)^{n_{3}} \prod_{j=n_{1}+n_{2}+n_{3}+1}^{n+1} F\left(\widetilde{v}_{j}^{*}\right)$.

Finally, from the first order condition with respect to $a_{3}$ bidders,

$$
\begin{aligned}
& c-\left[J\left(a_{3}\right) F\left(a_{3}\right)^{n_{1}+n_{2}}-n_{1} \int_{a_{1}}^{a_{2}} J(v) F(v)^{n_{1}-1} F\left(a_{2}\right)^{n_{2}} f(v) d v-\right. \\
& \left.\left(n_{1}+n_{2}\right) \int_{a_{2}}^{a_{3}} J(v) F(v)^{n_{1}+n_{2}-1} f(v) d v\right] F\left(a_{3}\right)^{n_{3}-1} \prod_{j=n_{1}+n_{2}+n_{3}+1}^{n+1} F\left(\widetilde{v}_{j}^{*}\right) \geq 0,
\end{aligned}
$$

or, after integration by parts,

$$
\begin{aligned}
c \geq & {\left[J\left(a_{1}\right) F\left(a_{1}\right)^{n_{1}} F\left(a_{2}\right)^{n_{2}}+\int_{a_{1}}^{a_{2}} J^{\prime}(v) F(v)^{n_{1}} F\left(a_{2}\right)^{n_{2}} d v+\right.} \\
& \left.\int_{a_{2}}^{a_{3}} J^{\prime}(v) F(v)^{n_{1}+n_{2}} d v\right] F\left(a_{3}\right)^{n_{3}-1} \prod_{j=n_{1}+n_{2}+n_{3}+1}^{n+1} F\left(\widetilde{v}_{j}^{*}\right) .
\end{aligned}
$$

From (17) and (18),

$$
J\left(a_{1}\right) F\left(a_{1}\right)^{n_{1}-1}\left[F\left(a_{2}\right)-F\left(a_{1}\right)\right] \geq \int_{a_{1}}^{a_{2}} J^{\prime}(v) F(v)^{n_{1}} d v .
$$


The B.E. Journal of Theoretical Economics, Vol. 9 [2009], Iss. 1 (Advances), Art. 24

with equality if $a_{1}>v_{l}$. Multiply both sides with $F\left(a_{1}\right)$. Now, either $F\left(a_{1}\right)=$ 0 or the above inequality holds as an equality. In either case,

$$
J\left(a_{1}\right) F\left(a_{1}\right)^{n_{1}}=\frac{F\left(a_{1}\right)}{F\left(a_{2}\right)-F\left(a_{1}\right)} \int_{a_{1}}^{a_{2}} J^{\prime}(v) F(v)^{n_{1}} d v .
$$

Adding $\int_{a_{1}}^{a_{2}} J^{\prime}(v) F(v)^{n_{1}} d v$ to both sides,

$$
J\left(a_{1}\right) F\left(a_{1}\right)^{n_{1}}+\int_{a_{1}}^{a_{2}} J^{\prime}(v) F(v)^{n_{1}} d v=\frac{F\left(a_{2}\right)}{F\left(a_{2}\right)-F\left(a_{1}\right)} \int_{a_{1}}^{a_{2}} J^{\prime}(v) F(v)^{n_{1}} d v .
$$

Similarly, from (18) and (19), we have

$$
\begin{aligned}
& J\left(a_{1}\right) F\left(a_{1}\right)^{n_{1}}+\int_{a_{1}}^{a_{2}} J^{\prime}(v) F(v)^{n_{1}} d v \\
\geq & \frac{\int_{a_{2}}^{a_{3}} J^{\prime}(v) F(v)^{n_{1}+n_{2}} d v}{F\left(a_{2}\right)^{n_{2}-1}\left[F\left(a_{3}\right)-F\left(a_{2}\right)\right]}>\frac{F\left(a_{2}\right) \int_{a_{2}}^{a_{3}} J^{\prime}(v) F(v)^{n_{1}} d v}{F\left(a_{3}\right)-F\left(a_{2}\right)},
\end{aligned}
$$

where the strict inequality follows from the fact that $F(v)$ is larger than $F\left(a_{2}\right)$ on $\left[a_{2}, a_{3}\right]$. Together with equality (20), this last inequality yields

$$
\frac{\int_{a_{1}}^{a_{2}} J^{\prime}(v) F(v)^{n_{1}} d v}{F\left(a_{2}\right)-F\left(a_{1}\right)}>\frac{\int_{a_{2}}^{a_{3}} J^{\prime}(v) F(v)^{n_{1}} d v}{F\left(a_{3}\right)-F\left(a_{2}\right)}
$$

or,

$$
\frac{\varphi\left(x_{2}\right)-\varphi\left(x_{1}\right)}{x_{2}-x_{1}}>\frac{\varphi\left(x_{3}\right)-\varphi\left(x_{2}\right)}{x_{3}-x_{2}},
$$

where $x_{i}=F\left(\widetilde{v}_{i}^{*}\right)$ and $\varphi(x)=\int_{0}^{F^{-1}(x)} J^{\prime}(v) F(v)^{n_{1}} d v$. Now notice that,

$$
\varphi^{\prime}(x)=\frac{J^{\prime}\left(F^{-1}(x)\right) F\left(F^{-1}(x)\right)^{n_{1}}}{f\left(F^{-1}(x)\right)}>0,
$$

and $\varphi^{\prime \prime}(x) \geq 0$ (since $J^{\prime}(v) \frac{F(v)}{f(v)}$ is weakly increasing), which contradicts (21).

Proof of Proposition 4. Suppose that in the optimal auction $k$ bidders have the cutoff $a$ and $n-k$ bidders have the cutoff $b$, where $1 \leq k \leq n-1$ 
Celik and Yilankaya: Optimal Auctions with Simultaneous and Costly Participation

and $v_{l} \leq a<b \leq v_{h}$. The following first order conditions must be satisfied: ${ }^{54}$

$$
\begin{gathered}
J(a) F(a)^{k-1} F(b)^{n-k}-c \geq 0, \\
J(b) F(b)^{n-1}-F(b)^{n-k-1} \int_{a}^{b} J(v) d F(v)^{k}-c \leq 0 .
\end{gathered}
$$

Combining these, and using integration by parts,

$$
J(a) F(a)^{k-1}(F(b)-F(a)) \geq \int_{a}^{b} J^{\prime}(v) F(v)^{k} d v .
$$

We will show the existence of $r \geq 0$ and $c^{e}$ such that there is an equilibrium of the second price auction with reserve price $r$ and effective participation cost $c^{e}$ in which $k$ (respectively, $n-k$ ) bidders participate iff their valuations are greater than $a$ (respectively, $b$ ), and all the participating bidders bid their valuations. ${ }^{55}$ We only need to check (the rest is standard, see, for example, Tan and Yilankaya, 2006) that $k$ bidders who have $a$ as their cutoffs have nonnegative (zero if $a>v_{l}$ ) expected payoffs when their valuations are $a$, and that the remaining bidders have nonpositive (zero if $b<v_{h}$ ) expected payoffs when their valuations are $b$ :

$$
\begin{gathered}
(a-r) F(a)^{k-1} F(b)^{n-k}-c^{e} \geq 0 . \\
F(b)^{n-k-1}\left((b-r) F(a)^{k}+\int_{a}^{b}(b-v) d F(v)^{k}\right)-c^{e} \leq 0,
\end{gathered}
$$

or, after using integration by parts,

$$
F(b)^{n-k-1}\left((a-r) F(a)^{k}+\int_{a}^{b} F(v)^{k} d v\right)-c^{e} \leq 0 .
$$

(25), with equality if $a>v_{l}$, and (26), with equality if $b<v_{h}$, have an

\footnotetext{
${ }^{54}$ Note that these conditions must be satisfied even in the constrained problem where two distinct cutoffs are used (with $k$ bidders using the smaller one), and the only choice variables are the magnitudes of these cutoffs. Therefore, we are also proving the claim in the text that an anonymous SPA can be used to improve over the optimal symmetric auction whenever our sufficient condition for asymmetry is satisfied (Proposition 1).

${ }^{55}$ The following will be true for $r$ and $c^{e}$ we find: $0 \leq c^{e}, r, c^{e}+r \leq a$.
} 
The B.E. Journal of Theoretical Economics, Vol. 9 [2009], Iss. 1 (Advances), Art. 24

admissible solution in $r$ and $c^{e}$, i.e., with $r \geq 0$ iff $^{56}$

$$
a F(a)^{k-1}(F(b)-F(a)) \geq \int_{a}^{b} F(v)^{k} d v .
$$

Since $a \geq J(a)$ and $J^{\prime}(v) \geq 1$ (because $\frac{1-F(v)}{f(v)}$ is decreasing), (24) implies (27), proving the result.

\section{References}

[1] Ayres, I. and P. Cramton (1996), "Deficit Reduction Through Diversity: How Affirmative Action at the FCC Increased Auction Competition," Stanford Law Review 48, 761- 815.

[2] Bergeman, D. and J. Valimaki (2002), "Information Acquisition and Efficient Mechanism Design," Econometrica 70, 1007-1033.

[3] Campbell, C. M. (1998), "Coordination in Auctions with Entry," Journal of Economic Theory 82, 425-450.

[4] Compte, O. and P. Jehiel (2007), "Auctions and Information Acquisition: Sealed Bid or Dynamic Formats?" Rand Journal of Economics 38, 355372 .

[5] Cremer, J., Y. Spiegel and C. Z. Zheng (2007), "Optimal Search Auctions," Journal of Economic Theory 134, 226-248.

[6] Ehrman, C. and M. Peters (1994), "Sequential Selling Mechanisms," Economic Theory 4, 237-253.

[7] Engelbrecht-Wiggans, R. (1993), "Optimal Auctions Revisited," Games and Economic Behavior 93, 227-239.

[8] Harstad, R. M. (1990), "Alternative Common-Value Auction Procedures: Revenue Comparison with Free Entry," Journal of Political Economy 98, 421-429.

[9] Levin, D. and J. L. Smith (1994), "Equilibrium in Auctions with Entry," American Economic Review 84, 585-599.

\footnotetext{
${ }^{56}$ If we do not impose the requirement that $r \geq 0$, then a solution always exists, as long as $F(a)^{k-1} \neq 0$. Note that $F(a)^{k-1} \neq 0$ for the optimal auction, see (22).
} 
Celik and Yilankaya: Optimal Auctions with Simultaneous and Costly Participation

[10] Lu, J. (2009), "Auction Design with Opportunity Cost," Economic Theory $38,73-103$.

[11] Matthews, S. A. (1984), "Information Acquisition in Discriminatory Auctions," in Bayesian Models in Economic Theory, ed. by M. Boyer and R. E. Kihlstrom, Elsevier Science Publishers, New York.

[12] McAfee, R. P. and J. McMillan (1987), "Auctions with Entry," Economics Letters 23, 343-347.

[13] McAfee, R. P. and J. McMillan (1988), "Search Mechanisms," Journal of Economic Theory 44, 99-123.

[14] McAfee, R. P. and J. McMillan (1989), "Government Procurement and International Trade," Journal of International Economics 26, 291-308.

[15] Menezes, F. M. and P. K. Monteiro (2000), "Auctions with Endogenous Participation," Review of Economic Design 5, 71-89.

[16] Myerson R. B. (1981), "Optimal Auction Design," Mathematics of Operations Research 6, 58-73.

[17] Persico, P. (2000), "Information Acquisition in Auctions," Econometrica 68, 135-148.

[18] Samuelson, W. F. (1985), "Competitive Bidding with Entry Costs," Economics Letters 17, 53-57.

[19] Stegeman, M. (1996), "Participation Costs and Efficient Auctions," Journal of Economic Theory 71, 228-259.

[20] Tan, G. (1992), "Entry and R\&D in Procurement Contracting," Journal of Economic Theory 58, 41-60.

[21] Tan, G. and O. Yilankaya (2006), "Equilibria in Second Price Auctions with Participation Costs," Journal of Economic Theory 130, 205-219. 
Copyright of B.E. Journals of Theoretical Economics: Advances in Theoretical Economics is the property of Berkeley Electronic Press and its content may not be copied or emailed to multiple sites or posted to a listserv without the copyright holder's express written permission. However, users may print, download, or email articles for individual use. 